\title{
Esquemas alternativos de vacunación contra Haemophilus Influenza tipo b permiten disminuir costos brindando adecuada inmunogenicidad
}

Economisation of vaccination against Haemophilus influenzae type b: a randomised trial of immunogenicity of fractional-dose and two-dose regimens. Lagos R, Valenzuela MT, Levine OS et. al. Lancet. 1998; 351: 1472-76.

\author{
Objetivo \\ Identificar programas de vacunación más económicos, con regímenes \\ alternativos a la serie estándar de tres dosis.
}

\section{Diseño}

Ensayo clínico aleatorizado simple ciego con un seguimiento promedio de 13 meses.

\section{Lugar}

Cuatro centros de salud de cuidados ambulatorios en Santiago de Chile.

\section{Pacientes}

Se incluyeron 627 recién nacidos a término con un peso al nacer de 2.500 grs. o más que entrarían a un programa atención primaria en centros ambulatorios.

\section{Intervención}

Los bebés se asignaron aleatoriamente a uno de cuatro regímenes con una u otra vacuna Hib conjugada: PRP-T (polisacárido - toxoide tetánico) o Hib PRP-CRM197 (oligosacárido - toxoide diftérico), para un total de 8 grupos. Los regímenes incluyen: a) Tres dosis completas, b) Tres dosis fraccionadas de la mitad de una dosis completa, c) Tres do- sis fraccionadas de un tercio de una dosis completa (2, 4 y 6 meses), y d) Dos dosis completas (4 y 6 meses).

\section{Medición de resultados principales}

El resultado principal fue la proporción de bebés con anticuerpos anti poliribosil-ribitol-fosfato en suero (PRP, polisacárido capsular del tipo b) con concentración de $0.15 \mu \mathrm{g} / \mathrm{ml}$ o más, a los 8 meses de edad.

\section{Resultados Principales}

El 93\% (IC 95\% 84-98) de bebés vacunados con tres dosis completas de PRP-T o PRP-CRM197 alcanzaron concentraciones anti-PRP de $0.15 \mu \mathrm{g} / \mathrm{mL}$ o más en la edad de 8 meses. La tasa de respuesta con regímenes de dosis fraccionadas fue del $91 \%$ (IC95 83-96) para los de la mitad de la dosis, y del 100\% (IC95\% 95-100) para bebés inmunizados con un tercio de dosis. De los bebés vacunados con dos dosis completas de PRP-T o PRPCRM197, el 99\% (IC95\% 93-100) y el 87\% (IC95\% 77-93) alcanzó concentraciones anti PRP adecuadas, respectivamente.

\section{Conclusiones}

El 91 al 100\% de los bebés inmunizados con dosis fraccionadas de Hib conjugada desarrolló concentraciones protectoras de anticuerpos. El regimen de dos dosis completas también es una opción válida.

\section{COMENTARIO}

Los resultados de este estudio de bioequivalencia *, muestran que regímenes alternativos con dosis fraccionadas (2, 4 y 6 meses) y dos dosis completas (4 y 6 meses), de las vacunas Hib conjugadas PRP-T y PRP-CRM197, logran niveles satisfactorios de anticuerpos en el $87-100 \%$ de los bebés inmunizados a los 8 meses de edad. La seroprotección obtenida con la vacuna PRP-T en esta investigación, es consistente con un estudio sueco que mostró que dos dosis de $7,5 \mu \mathrm{g}$ a los 3 y 5 meses de edad, produjeron una respuesta equivalente a dos dosis completas de $15 \mu \mathrm{g}(1)$. La vacuna Hib conjugada es la que mayor costo representa al sistema de salud chileno.(2), en comparación con las otras vacunas del Programa Ampliado de Inmunización administradas en los dos primeros años de vida. Por lo tanto, estos regímenes alternativos representan una posible vía de disminución de costos manteniendo la eficacia inmunogénica. Esta situación debería considerarse en países en desarrollo en los cuales no se logra una cobertura satisfactoria por limitaciones económicas. Esto puede implicar consecuencias muy graves tales como aumento de incidencia de meningitis, epiglotitis y neumonía, entre otras manifestaciones sistémicas (3), que aún con un manejo apropiado pueden dejar secuelas permanentes en los sobrevivientes (4). No obstante los resultados promisorios de este trabajo, quedan dudas sobre la garantía de un cambio en el régimen vigente de vacunación sobre la base del estudio de un solo lote de la vacuna. Por este motivo el comité de vacunación de la sociedad de enfermedades infecciosas Chilena propuso evaluar la inmunogenicidad de los restantes lotes de vacunas administrándolos en regímenes de dosis fraccionadas. De confirmarse los hallazgos de este estudio, se podrá entonces implementar un cambio de dosis que permita extender los beneficios de esta vacuna a un número mayor de niños sin aumentar los costos actuales del sistema.

*Ver Glosario

Dra. Nanci Giraudo

Unidad de Medicina Familiar y Preventiva. Hospital Italiano de Buenos Aires. 